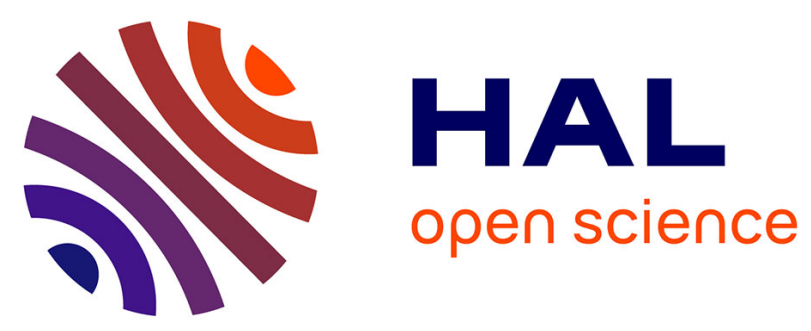

\title{
Potential drug-drug interactions and nephrotoxicity in hematopoietic stem cell transplant adult recipients during bone marrow transplantation unit stay
}

\author{
Lydia Sanchez, Astrid Bacle, Thierry Lamy, Pascal Le Corre
}

\section{- To cite this version:}

Lydia Sanchez, Astrid Bacle, Thierry Lamy, Pascal Le Corre. Potential drug-drug interactions and nephrotoxicity in hematopoietic stem cell transplant adult recipients during bone marrow transplantation unit stay. Cancer Chemotherapy and Pharmacology, 2019, 83 (5), pp.827-835. 10.1007/s00280019-03791-9 . hal-02061952

HAL Id: hal-02061952

https://hal-univ-rennes1.archives-ouvertes.fr/hal-02061952

Submitted on 13 Mar 2019

HAL is a multi-disciplinary open access archive for the deposit and dissemination of scientific research documents, whether they are published or not. The documents may come from teaching and research institutions in France or abroad, or from public or private research centers.
L'archive ouverte pluridisciplinaire HAL, est destinée au dépôt et à la diffusion de documents scientifiques de niveau recherche, publiés ou non, émanant des établissements d'enseignement et de recherche français ou étrangers, des laboratoires publics ou privés. 
Potential drug-drug interactions and nephrotoxicity in hematopoietic stem cell transplant adult recipients

1

2

3

4

5

6

7

8

9 during bone marrow transplantation unit stay

Lydia Sanchez $^{1-2}$, Astrid Bacle ${ }^{1,5,6}$, Thierry Lamy ${ }^{3,4}$, Pascal Le Corre ${ }^{1,5,6}$

1) Pôle Pharmacie, Service Hospitalo-Universitaire de Pharmacie, CHU de Rennes, 35033 Rennes, France.

2) Pharmacia Del Carre López, 48012 Bilbao, Espagna.

3) Pôle Médecines Spécialisées, Service d’Hématologie Clinique, CHU de Rennes, 35033 Rennes, France.

4) Univ Rennes 1, INSERM, EFS Bretagne, UMR U1236, F-35033 Rennes, France.

5) Laboratoire de Biopharmacie et Pharmacie Clinique, Faculté de Pharmacie, Université de Rennes 1, 35043 Rennes, France.

6) Univ Rennes, CHU Rennes, Inserm, EHESP, Irset - UMR_S 1085, F-35000 Rennes, France

Pr. Pascal Le Corre, corresponding author

Laboratoire de Biopharmacie et Pharmacie Clinique,

Faculté de Pharmacie

Université de Rennes 1, 35043 Rennes Cedex, France.

Phone +33223234872

Email pascal.le-corre@univ-rennes1.fr

ORCID : 0000-0003-4483-0957 


\section{Abstract}

Purpose: Studies have documented potential drug-drug interactions (pDDI's) occurring in cancer patients mainly with solid malignancies, either in the ambulatory or hospital settings. While hematopoietic stem cell transplant (HSCT) patients during their bone marrow transplantation unit (BMTU) stay have rather complex medical regimens combining chemotherapy, anti-infectious agents, immunosuppressive agents and supportivecare drugs, studies on potential DDI's are lacking.

Our objective was to evaluate the prevalence and the density of pharmacokinetic and pharmacodynamic potential DDI's, and the evolution of the renal function in hematopoietic stem cell transplant (HSCT) adult recipients during their BMTU stay.

Method: retrospective study in 31 adult patients consecutively admitted to the BMTU.

Results: Prevalence of pharmacokinetic interactions was 10-time lower than the pharmacodynamic interactions. The contra-indications were rare, and only of pharmacokinetic origin. The main drugs involved in pharmacokinetic DDI's were ciclosporine, methotrexate, esomeprazole, tramadol and vincristine. The median number of potential nephrotoxicity-related DDI's per patient was 7 and the median number of days during which nephrotoxicity-related DDI's potentially occurred was 77 days per patient. The decrease in glomerular filtration rate (GFR) throughout the BMTU stay (mean decrease of $13 \mathrm{ml} / \mathrm{min}$ ) was correlated with the number of days of potential nephrotoxic drug interactions.

Conclusions: Potential DDI's in HCST patients in BMTU were quite common. The DDI's from pharmacokinetic origin were less frequent, but of higher grade, than those of pharmacodynamic origin. The decrease in GFR suggests that the density of potential nephrotoxic drug interactions may be an issue to be considered in these patients. 
1

2

\section{Key words}

Pharmacokinetic, Pharmacodynamic, Drug-drug interactions, Hematopoietic stem cell transplant, Bone marrow transplantation unit stay, Nephrotoxicity. 


\section{Introduction}

Drug-drug interactions (DDIs) are quite common in the hematopoietic stem cell transplant (HSCT) patients given the number of drugs, and the rather complexity of medical regimens combining chemotherapy, antiinfectious agents, immunosuppressive agents and supportive-care drugs. Pharmacokinetic drug interactions involving inhibitors and inducers of P450 CYP3A4 can result in either increases or decreases in serum concentrations of drugs, potentially leading to enhanced toxicity or treatment failure [1,2]. However, drug-drug interactions involving membrane transporters should also be considered $[3,4]$. Pharmacodynamic drug interactions in HSCT patients are also likely but are less studied [5].

Numerous studies in cancer patients, mainly with solid malignancies, have shown that potential drug-drug interactions (pDDI's) were frequent, and that many were of clinical relevance [6-9].

Adverse drug reactions and drug interactions have been associated with unplanned admission in oncology patients in $13 \%$ and $2 \%$, respectively [10]. In a study achieved in hospitalized cancer patients with solid tumor, excluding patients in intensive care unit, potential drug interactions were detected in approximately $70 \%$ of the patients [11]. A study performed in oncological inpatients showed a prevalence of potential DDI's ranging from $33 \%$ to $81 \%$ depending on the software used for the analysis [12], and that most DDI's occurred between non-antineoplastic drugs (around $95 \%$ ). Although many interactions may be detected, they are not necessarily clinically significant. Potential drug interactions have been rated as severe or of major grade in $18.3 \%$ [11], $9 \%$ [6] and in $6 \%$ [13], respectively.

Several studies in hematological patients have have shown that potential drug-drug interactions (pDDI's) were frequent ranging from $50 \%$ to $100 \%$ of the patients [14-17], and that many were of pharmacokinetic origin [14-16]. The clinical relevance, variable according the studies, was estimated as major in $62 \%$ [15] and $82 \%$ [18] of the patients, and as moderate in $86 \%$ of the patients [14], $38 \%$ [15].

Patients hospitalized in BMTU for aplasia, after either allogeneic or autogeneic HSCT, frequently encounter several complications including infections, acute and chronic GVHD that remain major causes of mortality and morbidity [19]. These patients are at risk for drug-drug interactions because their treatments usually involve complex medical regimens including drugs with a narrow therapeutic index. To our knowledge there is no report on potential DDI's in HSCT patients in BMTU stay for aplasia.

Moreover, the frequent multiple comorbidities, including renal and liver dysfunction, poor nutritional status increase the risk of clinically significant drug interactions [2]. Pharmacodynamic interactions focused on kidneys may be considered in these patients since drug interactions are an important risk for the development of AKI [20], and because drugs with potential nephrotoxicity are administered to hematopoietic stem cell transplant recipients. Furthermore, it has been recently shown that decreased in GFR in adult HSCT patients was associated with higher risk of mortality [21]. 
The aim of our study was to evaluate the prevalence and the density of potential pharmacokinetic and

1

2

3

4

5

6

7

8

9

10

11

12

13

14

15

16

17

18

19

20

21

22

23

24

25

26

27

28

29

30

31

32

33

34

35

36

37

38

39

40

41

42

43

44

45

46

47

48

49

50

51

52

53

54

55

56

57

58

59

60

61

62

63

64

65 pharmacodynamic DDI's and the evolution of the renal function in HSCT adult recipients throughout their BMTU stay. 


\section{Materials and Methods}

This retrospective study has ben performed on 31 adult inpatients consecutively admitted to the BMTU. These patients had different hematologic malignancies, most of them undergoing HSCT at the Department of Clinical Hematology of the University Hospital in Rennes.

A clinical pharmacist collected the required data from the patient's medical records. These data were demographic characteristics (age and sex), the final diagnostic, the type of transplantation and the duration of the stay in the intensive care unit. All anticancer and non-anticancer treatments scheduled were recorded as a function of time during the stay. This allowed the determination of the frequency and of the density of potential DDI'S. The density was estimated by number of days per patient during which the interaction theoretically occurs throughout the stay (i.e., sum of the number of days during which a pair of interactant drugs are prescribed).

Drugs prescribed on as-needed basis were not recorded. The number of medications for each patient was determined by adding all pharmacological compounds. Each one was considered an individual medication for analysis, whatever the drug schedule (e.g., commercial combination of sulfamethoxazole and trimethoprim was counted as two drugs, and IV and oral immediate or controlled-release morphine was counted as one drug).

Theriaque software was use to screen the DDI's, and to classify the potential DDI's as pharmacokinetics or pharmacodynamics, or unknown origin. Pharmaceutical interactions as a result of chemical and/or physical incompatibility between drugs when mixed with each other and those involving food-related interactions, multivitamins and herbs were not analyzed as beyond the scope of the study. The potential DDI burden was defined as the number of potential DDIs identified for an individual subject during the stay in the intensive care unit.

The severity of the interaction, either from pharmacokinetic or pharmacodynamic origin, was rated according to the french classification reported in the RCP drug file that are used by the software Theriaque (available from Centre National Hospitalier d'Information sur le Médicament, CNHIM). These levels were: contraindication, association not recommended, use with caution and to be taken into account.

Contra-indication $(\mathrm{Cl})$ should be considered as major severity indicating that the interaction may be life threatening and/or require medical intervention to minimize or prevent serious adverse effects. It has an absolute character and should not be transgressed.

Association not recommended (ANR) should be considered as moderate severity with an interaction that may result in an exacerbation of the patient's conditions and/or require modification on therapy. It has to be avoided for most of the times, excepted after evaluation of the benefice/risk in the patient, and requires a close follow up of the patient.

Use with caution (UWC) should be considered as minor severity indicating that the interaction would have limited clinical effects (manifestations may include an increase in the frequency or severity of side effects but generally would not require major modification of therapy). The association is possible as soon as some 
recommendations are considered, especially at the beginning of the treatment (dose adaptation, increase in clinical and/or biological survey).

To be taken into account (TBTIA) signifies that the risk of DDI' exists, usually corresponding to an addition of side effects. For these DDI's there is no practical recommendation to be proposed. The physician has to weight the opportunity to use the drug combination.

The term 'potential DDI' refers to the theoretical possibility that a partBMTUlar drug alters the intensity of the pharmacological effect of another drug used by the same patient, thereby increasing or reducing the therapeutic effect and/or eliciting adverse reactions or responses other than those originally stemming from the drugs. The impact of these interactions on the clinical status of the patient was not reported or observed in this study.

All co-administered drugs were included in potential DDI identification. DDIs with over-the-counter drugs were not investigated in this study as the patients used only prescribed drugs.

The evolution of the GFR was performed throughout the BMTU stay by collecting serum creatinine values before (7-days before), at the beginning of the stay (d-0), in the middle of the stay (d-middle), at the end of the stay (d-last), and after the stay (7-days after). Renal function was evaluated by measurement of GFR according to the CKD-EPI formula (Chronic Kidney Disease-Epidemiology Collaboration). According to the CKD-EPI equation, GFR $=141 \times \min (\mathrm{Scr} / \mathrm{k}, 1)^{\alpha} \times \max (\mathrm{Scr} / \mathrm{k}, 1)^{-1.209} \times 0.993^{\mathrm{Age}} \times 1.018$ [if female] ${ }_{1} 1.159$ [if black], where Scr is serum creatinine, $\mathrm{k}$ is 0.7 for females and 0.9 for males, $\alpha$ is -0.329 for females and -0.411 for males, min indicates the minimum of Scr/kor 1, and max indicates the maximum of Scr/k or 1 [22] .

These data were used to study the evolution of renal function before and after the BMTU stay. Patients were classified into 5 stages of increasing severity as assessed by glomerular filtration rate (GFR) according to the Kidney Disease Outcomes Quality Initiative [23], before and after the BMTU stay.

Furthermore, we searched for a link between a deterioration of renal function and the density of potential DDI's focused on the kidneys by plotting the decrease in GFR as a function of the number of days of nephrotoxic drug interaction. 


\section{Results}

\section{Patient characteristics}

Demographic and clinical characteristics of the patients are summarized in Table 1. The median age was 52 years (range 19 to 66). The majority of the patients underwent allogenic BMT (70\%). The most frequent diagnosis was acute myeloid leukemia (AML). The median duration of aplasia was 19 days (range 5 to 56).

\section{Administered drugs}

The median number of anticancer and non-anticancer systemic drug administered during the stay was 2 (range 1 to 6 ) and 22 (range 6 to 29), respectively.

\section{Potential pharmacokinetic drug-drug interactions}

The characteristics of the drug-drug interactions are summarized in Table 2. A total of 66 pharmacokinetic potential DDI's and 729 pharmacodynamic potential DDI's were identified during the BMTU stay.

The number of pharmacokinetic interactions was 10-time lower than the pharmacodynamic interactions (66 vs. 729). The contra-indications were rare and only of pharmacokinetic origin. The more severe DDI's including contra-indications and association not recommended represented $24 \%$ of the pharmacokinetic DDI's; and were not observed for pharmacodynamic DDI's. The pharmacokinetic interactions were mainly in the group « use with caution » while the pharmacodynamic interactions were essentially of lower intensity (93\% in the group "to be taken into account»). As a whole the mean number of DDI's (pharmacokinetic plus pharmacodynamic) per patient was 25.6 throughout the stay in the intensive care unit.

The density of interaction was estimated by the duration of the interaction period. As a mean, the density was 172 days of interactions per patient, mainly from pharmacodynamic origin, and in the group « to be taken into account $»$.

The main pharmacokinetic interactions are presented in Table 3. The main drugs involved in the DDI's as objet drug were ciclosporine, methotrexate, esomeprazole, tramadol and vincristine. Among these, the most frequent interaction was between ciclosporine and fluconazole.

\section{Potential pharmacodynamic drug-drug interactions}

The main pharmacodynamic interactions were related to sedation (39.2\%) and nephrotoxicity (31.2 \%). The remaining pharmacodynamic interactions were hyper-kaliemia (2.9\%), ototoxicity (1.8\%) and hypo-kaliemia (1.2\%).

The median number of potential nephrotoxicity-related DDI's per patient was 7 (range 0 to 18). The median number of days during which nephrotoxicity-related DDI's potentially occurred was 77 days per patient (range 0 to 165 days). The drugs that were mainly involved in the pharmacodynamic nephrotoxicity-related potential DDI's were in descending order : colistine sulfate, ciclosporine, acyclovir, valaciclovir, amikacine, methotrexate and vancomycin. 


\section{Evolution of GFR throughout the BMTU stay}

Before BMTU stay, the majority of patients were in stage 1 (GFR $>90 \mathrm{ml} / \mathrm{min} / 1.73 \mathrm{~m}^{2}$ ) of chronic renal disease (61.2\%). Just over $25.0 \%$ were in stage 2 (GFR between $60-89 \mathrm{ml} / \mathrm{min} / 1.73 \mathrm{~m}^{2}$ ) and the rest of the population (12.9\%) was in stage 3 (GFR between $30-59 \mathrm{ml} / \mathrm{min} / 1.73 \mathrm{~m}^{2}$ ). After the BMTU stay, $32.3 \%$ of the patients were in stage 1 . The majority of the patients (35.5\%) were in stage 2, and $25.8 \%$ and $6.5 \%$ were in stage 3 and 4 , respectively (Figure 1). No significant difference was found between stages of renal failure before and after BMTU stay (Wilcoxon signed rank test: $p$-value $=0.86$ ).

The evolution of GFR throughout the BMTU stay presented in Figure 2 indicated that a decrease in renal function occurred with a mean decrease of $13 \mathrm{ml}$ in GFR. This decrease was significant (Wilcoxon signed rank test: $\mathrm{p}$-value $=0.011$ ).

There was a strong correlation between the variation in GFR, estimated by the difference before and after BMTU stay, and the number of days with potential nephrotoxic interaction (Pearson coefficient $r_{p}=-0.61$ ). These results are shown in Figure 3.

\section{Discussion}

Studies of potential DDI's have been carried out in different population of patients in the area of oncology and haematology, either in hospitalized or in ambulatory patients. However, to our knowledge, our study is the first performed in patients during aplasia hospitalized in BMTU.

\section{Prevalence of potential DDI's}

The results of this retrospective study showed that $33 \%$ the patients encountered 17 potential DDI's of the higher grades ( $\mathrm{Cl}$ and $\mathrm{ANR})$ so that the mean prevalence of the most severe DDI's (Cl and ANR) was of 0.61 interaction per patient (Table 3). Most of the patients encountered the least significant potential DDI's : UWC $(87 \%)$ and TBTIA (100 \%) with a mean rate of 3.1 and 22.5 DDI's per patient, respectively.

The frequency of potential DDI reported in our study is lower than those reported in patients with haematologic malignancies where major and moderate DDI's were recorded in $63 \%$ of the patients [15]. In a study performed at the time of the conditioning for BMT, $60 \%$ of the patients were shown to have at least one potential DDI with a median score of 2 ; and a moderate severity in $86 \%$ of the cases [14]. In a systematic review, it was shown that the frequency of potential DDIs varied from $12 \%$ to $63 \%$, and that the variability depended mainly on the type of study population [8]. The study design, the methodology (prospective vs. retrospective), the method of DDI screening and detection are also factors that may explain the high variability in DDI frequency [15].

in BMTU patients, outside the cancer area, studies showed that $54 \%$ to $79.5 \%$ of patients were exposed to at least one potential DDI [24-26].

On the whole, our results are quite difficult to compare since they have been obtained in a population of patients in which potential DDI's have not yet been studied. Furthermore, the heterogeneity in the data 
reported in the literature is quite high given the fact that different detection software programs are used with different ratings for DDIs.

\section{Density of potential DDI's and nephrotoxicity}

The vast majority of the potential DDI's were of pharmacodynamic origin (92\%) while of low severity (UWC and TBTIA). The pharmacokinetic DDI presented a rather different pattern with $24 \%$ rated as higher grades. These results suggest that the attention of health care professionals should be directed mainly towards potential pharmacokinetic DDI's in this subset of patients with hematological malignancies during the BMTU stay.

However, if some interactions are of low grade, it should be noticed that their density is quite high especially for pharmacodynamic DDI's. Indeed, the mean number of DDI rated as TBTIA was 25.6 per patient.

The density of DDI's can also be estimated by the period of time during which a patient is the subject of the interactions. Indeed, besides the number of interactions, the period of time during which the drugs interact is of interest since the longer the period, the higher the potential negative consequence may be. Data in Table 2 showed that the number of days with interactions was quite significant with a mean of 150 days with interactions rated TBTIA per patients (the mean length of stay was 19 days).

These data suggest that interactions of low grade may be considered if they are focused on a specific organ or tissue (e.g., liver, kidney and brain).

Although the pharmacodynamic DDI's were rated as from minor importance (cf Table 1), their density should be considered. Among the pharmacodynamic potential DDI's, those directed towards the kidneys should be considered given their frequency (around $30 \%$ of all pharmacodynamic DDI's) and the fact that this organ in readily involved in drug elimination. In our study, the drugs most frequently involved in potential nephrotoxicity (based on the density of interaction) were in decreasing order : colistin (32.5\%), ciclosporin (31.7\%), aciclovir (20\%) and valaciclovir (8.5\%). This suggests that a close therapeutic drug monitoring (TDM) of theses drugs should be performed. TDM is usual for calcineurin inhibitors but should also be useful for antiinfective drugs.

While variable between patients, the mean number of potential nephrotoxicity-related DDI's $(n=7)$, and the corresponding mean cumulative number of days per patient ( $n=77$ days) suggest that nephrotoxicity may be an issue.

Indeed, we noticed a significant decrease in renal function throughout the BMTU stay (mean decrease of 13 $\mathrm{ml} / \mathrm{min}$ in GFR, Figure 2). The decrease in renal function was also illustrated by shifts in renal stages of the patients : from stage 1 before BMTU stay to stage 2 or 3 after BMTU stay. This decrease in GFR may be related to stage 1 or to subclinical AKI.

It should be mentioned that AKI develops in the acute phase of HCT, and that these patients are at risk of developing CKD. This phenomenon has already been described, and is refered the "post-HCT CKD" [27], but remains not very well known by hematologists and nephrologists. Such deterioration in renal function may be of concern because patient survival may be limited by treatment-related toxicities including acute kidney injury (AKI) that can arise up to $70 \%$ of the patients after transplantation (28). 
Non-recovery of kidney function following an episode of AKI is a major problem, and the severity and the number of episodes of AKI are associated with the development of incident CKD and ESRD (29). Furthermore, a recent prospective study in long-term survival HCT patients reported a dramatic decline in glomerular filtration rate over the first year post-HCT associated with a higher risk of mortality [21].

We also found that the decrease in renal function was strongly correlated with the number of days of treatment with potential nephrotoxic DDI (Figure 3).

The current study clearly showed that the addition of several nephrotoxic pharmacodynamic DDI's of low grade may have a negative outcome on the renal function. This should not be unlikely since the cumulative prescription of drugs with potential nephrotoxic drug interactions was quite common. A median number of 7 drugs with potential nephrotoxic interaction per patient was noticed, leading to a median number of 77 days with nephrotoxic interactions per patient. Such a feature is not unlikely since patients hospitalized in BMTU are often exposed to multiple concurrent nephrotoxins [30].

This density in nephrotoxic interaction may contribute to morbidity and mortality in these patients. It should be noticed that this comment is speculative and would deserve to be studied prospectively in a larger population of patients.

Given their number and density, potential nephrotoxicity-related DDI's may be considered as a covariate in future studies in order to identify patients with higher risk of kidney injury and to target potentialinterventions (e.g., use of angiotensin-converting enzyme inhibitors or angiotensin blockers).

\section{Pattern of potential pharmacokinetic DDI's}

On a whole the pharmacokinetic DDI's were less frequent than the pharmacodynamic DDI's. However, when considering the higher grades of severity, the pharmacokinetic DDI's were more prevalent (84 \%). This prevalence was higher than those reported in previous studies that were around $70 \%$ [15] and $55 \%$ [16]. The most commonly used therapeutic classes for nephrotoxicity are the antibiotics, anti-rejection medications, antiviral agents, non-steroidal anti-inflammatory agents, anti-ulcer agents and chemotherapy [31].

In our population of patients, the two drugs most involved in pharmacokinetic DDI were ciclosporine and methotrexate interacting principally with prophylactic treatment of bacterial and fungal infections (Table 3).

As calcineurin inhibitors are the main component of immunosuppressive prophylaxis against GVHD in allogeneic HSCT recipients, attention should be paid to these DDI's. With regard to ciclosporine, the main drug involved in DDI was fluconazole (in 22 patients) which is a moderate inhibitor of CYP3A4 and CYP2C9 and a strong inhibitor of CYP2C19 [32]. The consequence was an increase in ciclosporine levels above the range (100$300 \mathrm{ng} / \mathrm{ml}$ ) for $81.6 \%$ of measured trough levels during the BMTU stay. Outside the aplasia period, ciclosporin through levels were out-of-range in $18.8 \%$ of measurements. These significant variations suggest the need of a close therapeutic drug monitoring in these patients with a more frequent dosing adjustment and a carefull analysis of DDI's. 
The prevention of potential pharmacokinetic and pharmacodynamic DDI's in cancer patients is not a simple task given that complex medical regimen are administered including drugs with narrow therapeutic index, especially in HSCT recipients, and given the rather complexity of DDI's that can involve interactions at the metabolic level (mainly CYP450 enzymes) and/or at the membrane transporter level (2). Another difficulty may also arise from the fact that different databases can report differently potential interaction between two drugs (12).

Moreover, it should be kept in mind that in case of detected DDI, choosing alternative options are often not possible so that treatment may need to be maintained and adjusted to minimize potential outcomes. Hence, such task would need a multiprofessional work among nurses, pharmacists and physicians, and implementing a comprehensive team approach aimed at updating treatment regimens and systematic analysing of potential drug interactions for every patient may be considered (2).

Since teams of health professionals are often not completely familiar with DDI's that can threaten patient's life, a thorough sensibilization of all health professionnals on the DDI pathway, and on the potential impact of these DDI's may be achieved to reduce preventable adverse outcomes related to DDI's.

As similar observational studies, our investigation has several limitations. This is a retrospective single-center study hindered by a small sample size. Since we did not have measured GFR available, we used serum creatinine to calculated GFR. Furthermore there was no follow-up of the renal function at a distance from BMTU discharge. Moreover, the real consequences of the DDI's have not been evaluated (a reason why we used the expression potential DDI). Based on these elements, care should be taken before extrapolating the findings. However, it should be noticed that studies evaluating the clinical consequences are quite scarce. Further studies on the epidemiology and the real clinical consequences of DDIs in HSCT patients should be performed with prospective multi-center studies that could help in developing preventive strategies.

\section{Conclusion}

The current study has shown that potential DDI's in HCST patients in BMTU were quite common. The DDI's from pharmacokinetic origin were less frequent, but of higher grade, than those of pharmacodynamic origin. The estimation of the density of potential pharmacodynamic DDI's showed that nephrotoxicity may be an issue since the decrease in GFR was correlated with the number of days with nephrotoxic interactions per patient.

A careful TDM of the most nephrotoxic drugs should be performed to avoid an impairment of the renal function since recent data in the literature have shown that a decrease of the GFR has been associated to higher risk of mortality in HSCT patient by 1-year after transplantation.

Given the potential risk of DDI's, health professional caring for patients in period of aplasia in the intensive care unit should be aware of these interactions, and screen all new medications against a full medication history to 
attempt to decrease their prevalence. An integrated health care team including physicians, pharmacists, nursing staff, and focusing on DDI's prevention could contribute to a more appropriate and safe use of drugs in patients undergoing BMT.

\section{Conflict of interest}

The authors declare that they have no conflict of interest.

\section{Compliance with ethical standards.}

The study was approved by the Institutionnal Ethical committee of our institution with a waiver of patient consent authorization ( $\mathrm{N}^{\circ}$ 18.76). Since the design of the study is retrospective no formal consent is required. 


\section{References}

1 - Leather HL (2004)

Drug interactions in the hematopoietic stem cell transplant (HSCT) recipient: what every transplanter needs to know.

Bone Marrow Transplant 33:137-52

2 - Glotzbecker B, Duncan C, Alyea E 3rd, Campbell B, Soiffer R. (2012).

Important drug interactions in hematopoietic stem cell transplantation: what every physician should know. Biol Blood Marrow Transplant 18:989-1006.

3 - Gay C, Toulet D, Le Corre P (2017)

Pharmacokinetic drug-drug interactions of tyrosine kinase inhibitors: A focus on cytochrome P450, transporters, and acid suppression therapy.

Hematol Oncol 35:259-280.

4 - Morival C, Oumari S, Lenglet A, Le Corre P (2017)

Clinical pharmacokinetics of oral drugs in the treatment of multiple myeloma.

Hematol Oncol 27. doi: 10.1002/hon.2485.

5 - Riechelmann RP, Saad ED (2006).

A systematic review on drug interactions in oncology.

Cancer Invest 24:704-12.

6 - Riechelmann RP, Tannock IF, Wang L, Saad ED, Taback NA, Krzyzanowska MK (2007)

Potential drug interactions and duplicate prescriptions among cancer patients.

J Natl Cancer Inst 99:592-600

7 - Riechelmann RP, Zimmermann C, Chin SN, Wang L, O'Carroll A, Zarinehbaf S, Krzyzanowska MK (2008) Potential drug interactions in cancer patients receiving supportive care exclusively.

J Pain Symptom Manage 35:535-43.

8 - Riechelmann RP, Del Giglio A (2009)

Drug interactions in oncology: how common are they?

Ann Oncol 20:1907-12

9 - Kannan G, Anitha R, Rani VN, Thennarasu P, Alosh J, Vasantha J, Martin JR, Uma MR (2011)

A study of drug-drug interactions in cancer patients of a south Indian tertiary care teaching hospital.

J Postgrad Med 57:206-10

10 - Miranda V, Fede A, Nobuo M, Ayres V, Giglio A, (2011)

Adverse drug reactions and drug interactions as causes of hospital admission in oncology.

J Pain Symptom Manage 42:342-53.

11 - Riechelmann RP, Moreira F, Smaletz O, Saad ED (2005)

Potential for drug interactions in hospitalized cancer patients.

Cancer Chemother Pharmacol 56:286-90.

12 - Fernández de Espinosa MÁ, Díaz Carrasco MS, Alonso Romero JL, de la Rubia Nieto A, Espuny Miró A (2015)

Potential drug-drug interactions in oncological adult inpatients at a Spanish hospital: epidemiology and risk factors.

Int J Clin Pharm. 37:1021-7

13 - Vecchia S, Orlandi E, Confalonieri C, Damonti E, Riva A, Sartori A, Cavanna L (2017)

Prevalence study on potential drug-drug interaction in cancer patients in Piacenza hospital's OncoHaematology department. 
14 - Guastaldi RB, Reis AM, Figueras A, Secoli SR (2011)

Prevalence of potential drug-drug interactions in bone marrow transplant patients. Int J Clin Pharm 33:1002-1009.

15 - Hadjibabaie M, Badri S, Ataei S, Moslehi AH, Karimzadeh I, Ghavamzadeh A (2013)

Potential drug-drug interactions at a referral hematology-oncology ward in Iran: a cross-sectional study.

Cancer Chemother Pharmacol 71:1619-27.

16 - Gholaminezhad S, Hadjibabaie M, Gholami K, Javadi MR, Radfar M, Karimzadeh I, Ghavamzadeh A (2014) Pattern and associated factors of potential drug-drug interactions in both pre- and early post-hematopoietic stem cell transplantation stages at a referral center in the Middle East.

Ann Hematol 93:1913-22.

17 - Schaefer L, Keinki C, Baron von Osthoff M, Huebner J (2018)

Potential Interactions of Medication Prescribed in Discharge Letters from a Clinic for Hematology and Oncology.

Oncol Res Treat 41:200-205. doi: 10.1159/000486161

18 - Trevisan DD, Silva JB, Oliveira HC, Secoli SR, Lima MH (2015)Prevalence and clinical significance of potential drug-drug interaction in hematopoietic stem cell transplantation

Cancer Chemother Pharmacol 75:393-400

19 - Arnaout K, Patel N, Jain M, El-Amm J, Amro F, Tabbara IA (2014)

Complications of allogeneic hematopoietic stem cell transplantation.

Cancer Invest 32:349-62.

20 - Hoste EA, Bagshaw SM, Bellomo R, Cely CM, Colman R, Cruz DN, et al (2015)

Epidemiology of acute kidney injury in critically ill patients: the multinational AKI-EPI study.

Intensive Care Med 41:1411

21 - Hingorani S, Pao E, Stevenson P, Schoch G, Laskin BL, Gooley T, McDonald GB (2018)

Changes in Glomerular Filtration Rate and Impact on Long-Term Survival among Adults after Hematopoietic Cell Transplantation: A Prospective Cohort Study.

Clin J Am Soc Nephrol. 2018 Apr 18

22 - Levey AS, Stevens LA, Schmid CH, Zhang YL, Castro AF 3rd, Feldman HI, Kusek JW, Eggers P, Van Lente F, Greene T, Coresh J; CKD-EPI (Chronic Kidney Disease Epidemiology Collaboration) (2009)

A new equation to estimate glomerular filtration rate.

Ann Intern Med 150:604-12

23 - Clinical Practice Guidelines For Chronic Kidney Disease: Evaluation, Classification and Stratification National Kidney Foundation, Kidney Disease Outcomes Quality Initiative (2002)

24 - Farzanegan B, Alehashem M, Bastani M, Baniasadi S (2015)

Potential drug-drug interactions in cardiothoracic intensive care unit of a pulmonary teaching hospital. J Clin Pharmacol 55:132-6.

25 - Uijtendaal EV, van Harssel LL, Hugenholtz GW, Kuck EM, Zwart-van Rijkom JE, Cremer OL, Egberts TC (2014)

Analysis of potential drug-drug interactions in medical intensive care unit patients.

Pharmacotherapy 34:213-9.

26 - Vanham D, Spinewine A, Hantson P, Wittebole X, Wouters D, Sneyers B (2017)

Drug-drug interactions in the intensive care unit: Do they really matter?

J Crit Care 38:97-103. 
27 - Ando M, Ohashi K, Akiyama H, Sakamaki H, Morito T, Tsuchiya K, Nitta K (2010)

Chronic kidney disease in long-term survivors of myeloablative allogeneic haematopoietic cell transplantation: prevalence and risk factors.

Nephrol Dial Transplant 25:278-82

28 - Kogon A, Hingorani S (2010)

Acute kidney injury in hematopoietic cell transplantation.

Semin Nephrol, 30 :615-26

29 - Binnenmars SH, Hijmans RS, Navis G, de Borst MH (2017)

Biomarkers of Renal Function: Towards Clinical Actionability.

Clin Pharmacol Ther, 102 :481-492

30 - Rewa O, Bagshaw SM (2014)

Acute kidney injury-epidemiology, outcomes and economics.

Nat Rev Nephrol, 10:193-207.

31 - Awdishu L, Mehta RL (2017)

The 6R's of drug induced nephrotoxicity.

BMC Nephrol. 18:124. doi: 10.1186/s12882-017-0536-3.

32 - U.S. Food and Drug Administration. Drug Development and Drug Interactions: Table of Substrates, Inhibitors and Inducers.

http://www.fda.gov/Drugs/DevelopmentApprovalProcess/DevelopmentResources/Drug/nteractionsLabeling/u cm093664.htm\#4. Accessed 30 may 2018. 
1

2

3

4

7

8

10

11

12

13

14

15

16

17

18

19

20

21

22

23

24

25

26

27

28

29

30

31

32

33

34

35

36

37

38

39

40

41

42

43

44

45

46

47

48

49

50

51

52

53

54

55

56

57

58

59

60

61

62

63

64

65 
Figure 1: Evolution of stage of chronic kidney disease in patients 7-days before and 7-days after the BMTU stay.

Figure 2: Figure 2: Box plot showing the distribution of glomerular filtration rate (GFR) 7-days before, at the beginning of the stay $(d-0)$, in the middle of the stay (d-middle), at the end of the stay (d-last), and after the stay (7-days after). ( $\diamond$ : average GFR).

Figure 3: Variation in GFR before and after BMTU stay as a function of the number of days with potential nephrotoxic DDI.

\section{Table 1. Patient characteristics}

Table 2. Prevalence (number of interactions per patient, \%) and density (number of days with interactions per patient, in days) of pharmacokinetic and pharmacodynamic potential DDI's classified by their severity grade.

Table 3. Main pharmacokinetic DDI's 
Table 1. Patient characteristics

Total number of patients

Median age $(Y)$, range

$52(19-66)$

Sex

Female

Male

Cancer type

AML

8

ALL

Myeloma

3

Myelodysplastic syndroma

5

CLL

Lymphoma

Myelofibrosis

Thymoma

CML

5

3

3

2

1

1

Graft

no 8

Allograft

22

Autograft

1

Duration of aplasia (days, median (range))

$19(5-56)$

No. of drugs prescribed per patient, median (range)

Non anticancer systemic drugs

$22(6-29)$

anticancer systemic drugs

$2(1-6)$

TPN drugs

$3(0-5)$

No of drug interactions per patients, median (range)

$16(0-46)$ 
Table 2. Prevalence (number of interactions per patient, \%) and density (number of days with interactions per patient, in days) of pharmacokinetic and pharmacodynamic potential DDI's classified by their severity grade.

\begin{tabular}{|c|c|c|c|c|c|c|}
\hline & \multicolumn{2}{|c|}{ Pharmacokinetic } & \multicolumn{2}{|c|}{ Pharmacodynamic } & \multirow{2}{*}{$\begin{array}{l}\text { Total } \\
\mathrm{N}\end{array}$} & \multirow{2}{*}{$\begin{array}{l}\text { Prevalence of interactions (\%) } \\
\text { number of interactions per patient }\end{array}$} \\
\hline & $\mathrm{N}$ & $\%$ & $\mathrm{~N}$ & $\%$ & & \\
\hline contra-indication & 2 & 3 & 0 & 0 & 2 & 0.06 \\
\hline association not recommended & 14 & 21 & 3 & 0 & 17 & 0.55 \\
\hline use with caution & 36 & 55 & 47 & 6 & 83 & 2.68 \\
\hline to be taken into account & 14 & 21 & 679 & 93 & 693 & 22.4 \\
\hline \multirow[t]{3}{*}{ total } & 66 & 100 & 729 & 100 & 795 & 25.6 \\
\hline & \multicolumn{2}{|c|}{ Pharmacokinetic } & \multicolumn{2}{|c|}{ Pharmacodynamic } & Total & Density of interactions \\
\hline & Days & $\%$ & Days & $\%$ & Days & number of days with interactions per patient (d) \\
\hline contra-indication & 5 & 1 & 0 & 0 & 5 & 0.16 \\
\hline association not recommended & 48 & 9 & 27 & 0.6 & 75 & 2.4 \\
\hline use with caution & 410 & 79 & 197 & 4 & 607 & 19.9 \\
\hline to be taken into account & 54 & 10 & 4593 & 95 & 4647 & 150 \\
\hline total & 517 & 100 & 4817 & 100 & 5335 & 172 \\
\hline
\end{tabular}



Table 3. Main pharmacokinetic interactions (\%).

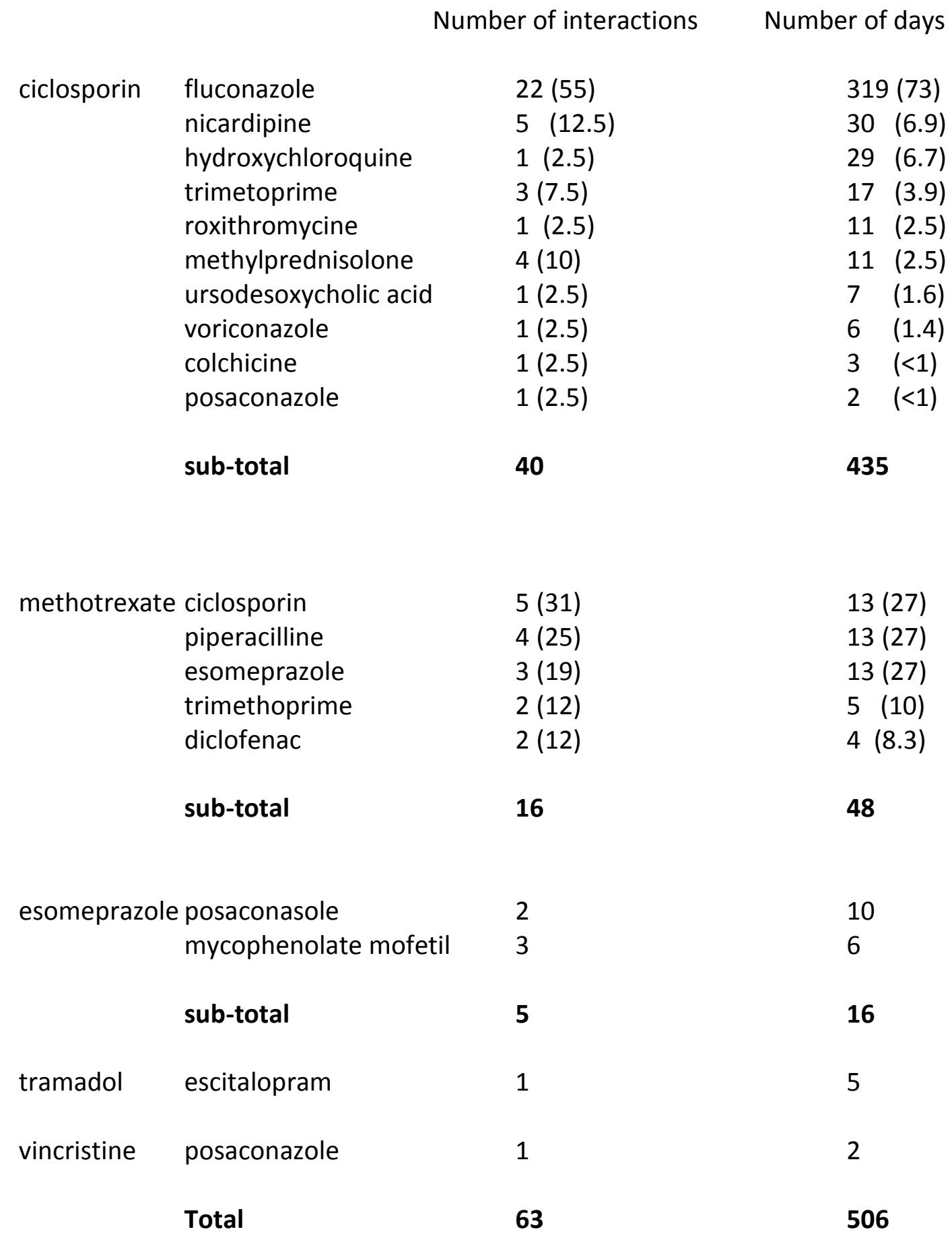


Figure 1: Evolution of stage of chronic kidney disease in patients 7-days before and 7-days after the BMTU stay.

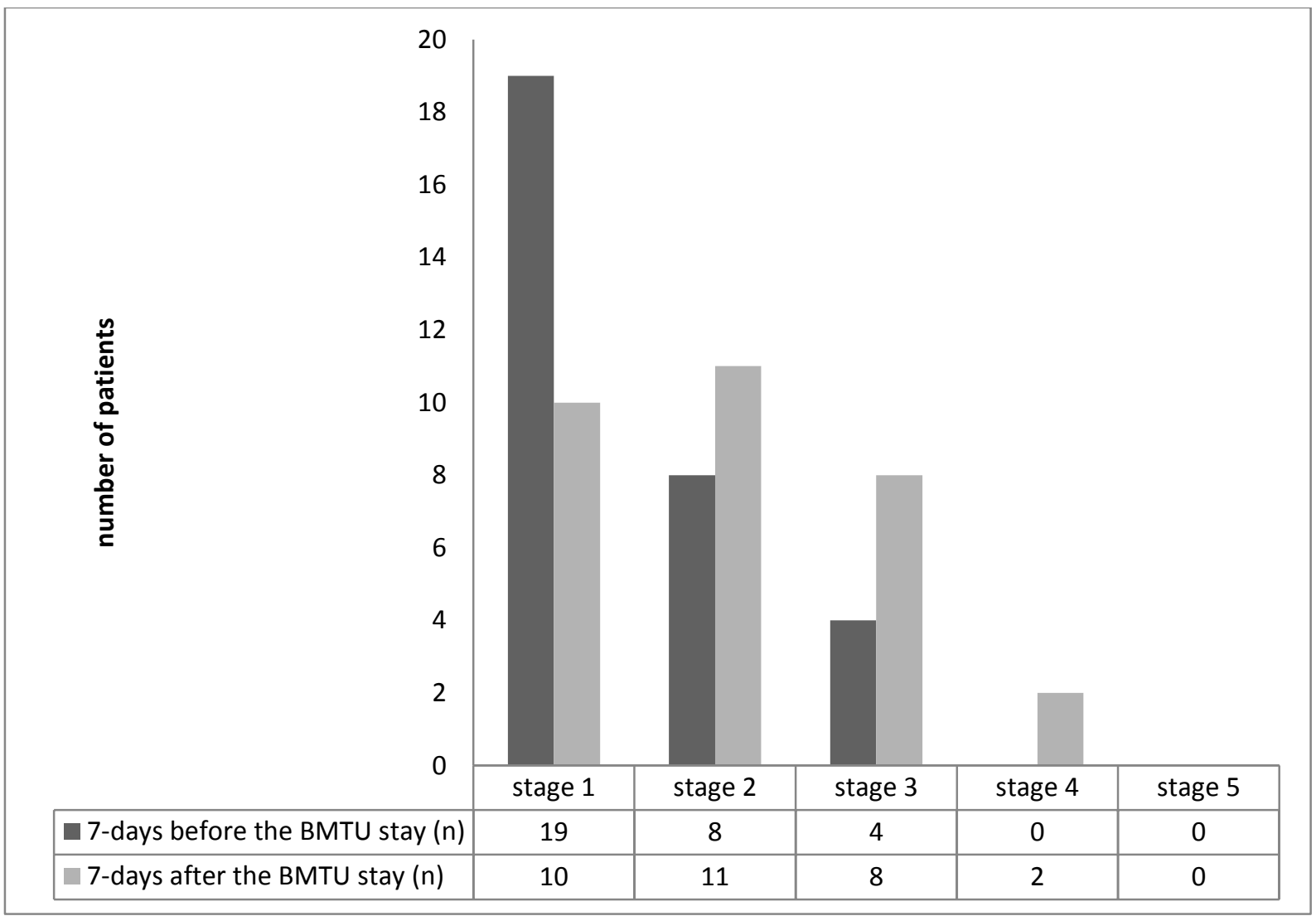


Figure 2: Box plot showing the distribution of glomerular filtration rate (GFR) 7-days before, at the beginning of the stay (d-0), in the middle of the stay (d-middle), at the end of the stay (d-last), and after the stay (7-days after) in patients in BMTU. ( $\diamond$ : average GFR).

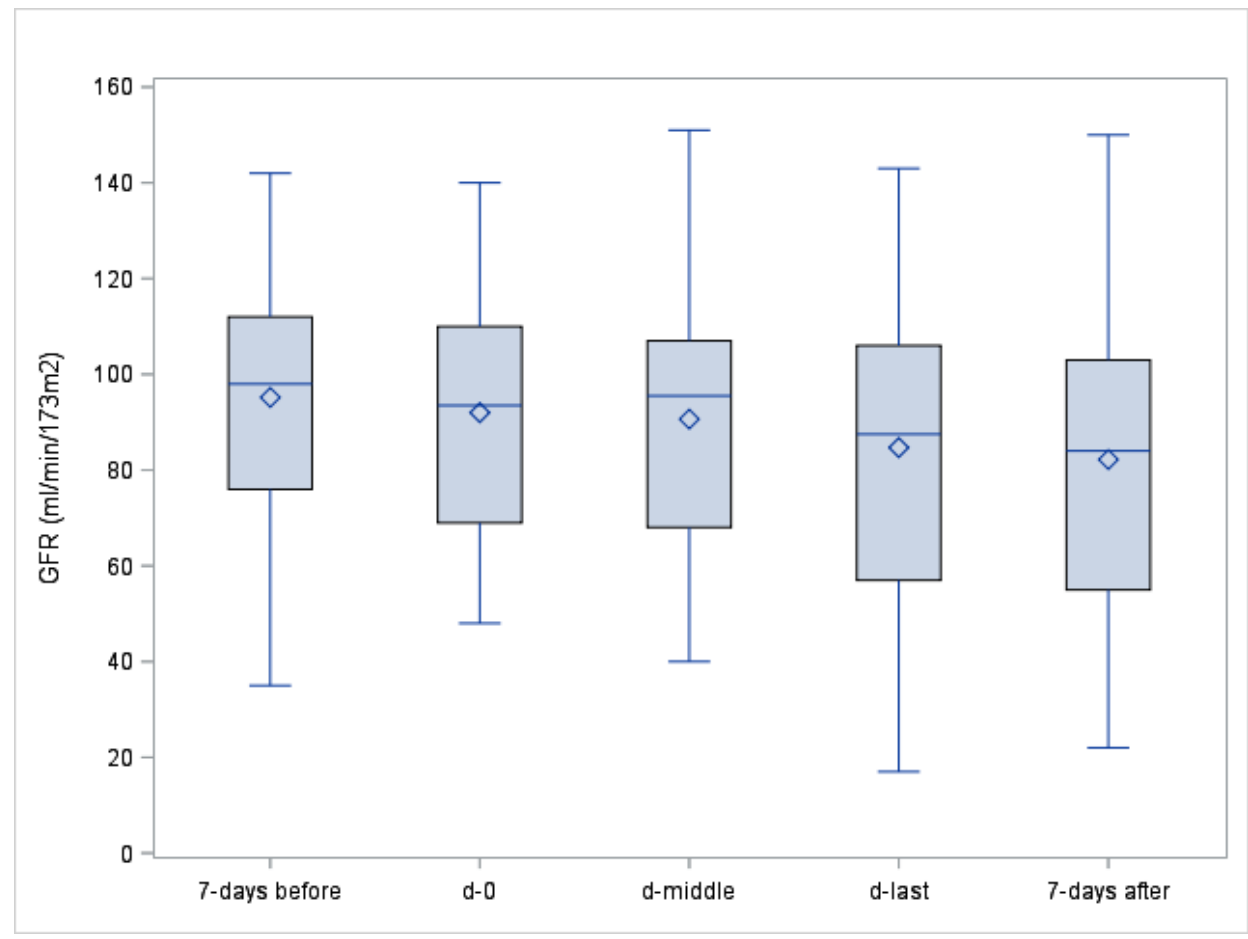

Box plot explanation: upper horizontal line of box, 75th percentile; lower horizontal line of box, 25th percentile; horizontal bar within box, median; upper horizontal bar outside box, 90th percentile; lower horizontal bar outside box, 10th percentile. 
Figure 3: Variation in GFR before and after BMTU stay as a function of the number of days with potential nephrotoxic DDI.

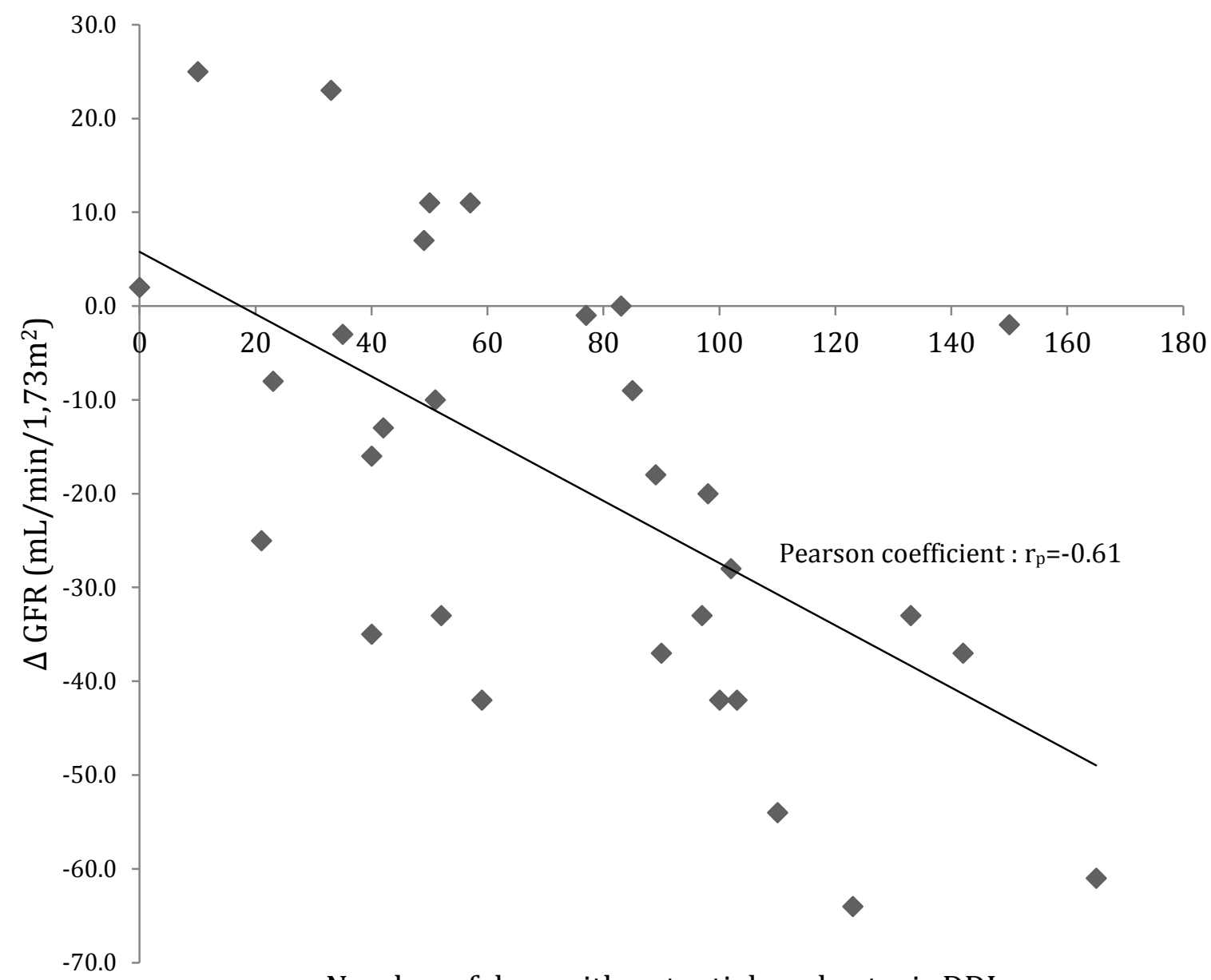

Number of days with potential nephrotoxic DDI 